\title{
BMJ Open Study protocol for a national cohort of adults focused on respiratory health: the American Lung Association Lung Health Cohort (ALA-LHC) Study
}

\author{
Paul A Reyfman (D) , ${ }^{1}$ Elizabeth Sugar, ${ }^{2}$ Heather Hazucha, ${ }^{3}$ Jenny Hixon, ${ }^{1}$ \\ Curt Reynolds, ${ }^{2}$ Sonali Bose, ${ }^{4}$ Mark T Dransfield, ${ }^{5}$ MeiLan K Han, ${ }^{6}$ \\ Raul San Jose Estepar, ${ }^{7,8}$ Mary B Rice, ${ }^{9,10}$ George R Washko, ${ }^{8,11}$ \\ Mercedes Carnethon, ${ }^{12}$ Ravi Kalhan (D) , ${ }^{1,12}$ American Lung Association Airways \\ Clinical Research Network
}

To cite: Reyfman PA, Sugar E, Hazucha $\mathrm{H}$, et al. Study protocol for a national cohort of adults focused on respiratory health: the American Lung Association Lung Health Cohort (ALA-LHC) Study. BMJ Open 2021;11:e053342. doi:10.1136/ bmjopen-2021-053342

- Prepublication history for this paper is available online. To view these files, please visit the journal online (http://dx.doi. org/10.1136/bmjopen-2021053342).

PAR and ES contributed equally.

Received 12 May 2021

Accepted 23 June 2021

Check for updates

(C) Author(s) (or their employer(s)) 2021. Re-use permitted under CC BY-NC. No commercial re-use. See rights and permissions. Published by BMJ.

For numbered affiliations see end of article.

Correspondence to

Dr Ravi Kalhan;

r-kalhan@northwestern.edu

\section{ABSTRACT}

Introduction The current framework for investigating respiratory diseases is based on defining lung health as the absence of lung disease. In order to develop a comprehensive approach to prevent the development of lung disease, there is a need to evaluate the full spectrum of lung health spanning from ideal to impaired lung health. The American Lung Association (ALA) Lung Health Cohort is a new, population-based, cohort study focused primarily on characterising lung health in members of the millennial generation without diagnosed severe respiratory disease. Participants will be enrolled for the baseline study visit starting in 2021, and funding will be sought to support future study exams as part of a longitudinal cohort study. This study will be crucial for developing a novel paradigm of lung health throughout the adult life course.

Methods and analysis This study will leverage the existing infrastructure of the ALA Airways Clinical Research Centers network to enrol 4000 participants between ages 25 and 35 years old at 39 sites across the USA between April 2021 and December 2024. Study procedures will include physical assessment, spirometry, chest CT scan, accelerometry and collection of nasal epithelial lining fluid, nasal epithelial cells, blood and urine. Participants will complete questionnaires about their sociodemographic characteristics, home address histories and exposures, work history and exposure, medical histories, lung health and health behaviours and activity.

Ethics and dissemination The study was approved by the Johns Hopkins Medicine Institutional Review Board. Findings will be disseminated to the scientific community through peerreviewed journals and at professional conferences. The lay public will receive scientific findings directly through the ALA infrastructure including the official public website. Deidentified datasets will be deposited to BioLINCC, and deidentified biospecimens may be made available to qualified investigators along with a limited-use datasets.

\section{INTRODUCTION AND RATIONALE}

Developing a robust approach to lung disease prevention requires a broad definition of lung health. ${ }^{1}$ While the respiratory research

\section{Strengths and limitations of this study}

- This is the first large, prospective US cohort study of respiratory health over the adult life course.

- Lung health outcomes are defined broadly and include respiratory symptoms, physiological lung function and CT scan-defined lung and airways injury.

- A wide array of biospecimens including blood, urine and nasal samples will be collected at the baseline assessment, which will allow for discovery of biomarkers of impaired respiratory health.

- Individuals will provide extensive information on modifiable exposures and risk factors (eg, lifetime air pollution, potentially noxious inhalation exposure such as marijuana and e-cigarettes, reduced physical activity and workplace exposures), which will be updated throughout the course of follow-up.

- Current funding supports only a cross-sectional examination and remote follow-up contacts; additional funding will be required for future in-person study exams and the ascertainment of long-term lung health outcomes.

community implicitly construes lung health as the absence of overt lung disease, the cardiovascular (CV) community has explicitly delineated a continuum ranging from ideal health to impaired health to established disease. According to this framework, $\mathrm{CV}$ health is composed of a set of factors, including several that directly occur along the causal pathway from health to disease. This paradigm of CV health serves as a foundation for developing preventive health measures by targeting risk factors for loss of health and recognition of measurable intermediate endotypes, such as heightened inflammation and hypercholesterolaemia. 
The American Lung Association Lung Health Cohort (ALA-LHC) Study is motivated by the conviction that a large, population-based cohort of young adults focused on the respiratory system is needed to define the constructs of of 'ideal' versus 'impaired' lung health. Previous studies of subclinical lung disease have examined healthy controls in disease-focused studies or used limited information about respiratory symptoms, physiology or imaging derived from cohort studies focused on other chronic conditions. Existing prospective cohort studies within the respiratory field have a maximum follow-up of 10 years and are focused on specific respiratory diseases, such as asthma and chronic obstructive pulmonary disease (COPD). Life course studies of lung health are necessary to describe how an individual progresses from ideal lung health to an intermediate phenotype of impaired lung health and ultimately to chronic lung disease.

We have developed a conceptual paradigm of lung health according to which there are two fundamental forms of impaired lung health: (1) impaired 'reserve' or lower peak lung function in young adult and (2) 'susceptibility' to impaired lung health or accelerated decline in lung function from young adulthood to middle age. The ALA-LHC will focus on modifiable exposures and clinical and behavioural risk factors in members of the millennial generation that we hypothesise are associated with impaired lung health reserve and on biomarkers of susceptibility to future lung disease. Identifying the factors associated with reserve and susceptibility will enhance the depiction of the continuum of lung health and will facilitate designing novel prevention strategies.

\section{Specific aims}

Our long-term goal is to determine factors evident in early adulthood that influence risk for future lung disease through detailed assessments of modifiable and non-modifiable environmental, clinical and behavioural risk factors and responses (physiological, imaging-based and biological including the nasal epithelial transcriptome). Our motivations were bolstered by a call from the National Heart, Lung, and Blood Institute (NHLBI) for the development of new epidemiology cohorts to address research hypothesis that were beyond the scope of existing cohorts. ${ }^{2}$ We have observed that impaired lung health (lower lung function, self-report of respiratory symptoms and lung abnormalities on chest CT scan) precedes the development of chronic lung disease. Therefore, the focus of the initial contact will be to establish the cohort, characterise their current lung health and test hypotheses about the risk factors and manifestations of impaired lung health in young adults. There are three specific aims:

1. Determine whether modifiable exposures and risk factors (lifetime air pollution, potentially noxious inhalational exposures such as marijuana and e-cigarettes and reduced physical activity) are associated with lower lung function and greater burden of respiratory symptoms in young adults.
2. Determine whether CT measurements of small airway and parenchymal lung abnormalities are associated with lower lung function and greater burden of respiratory symptoms in young adults.

3. Collect and store biospecimens for future analyses to determine the association between the nasal respiratory epithelial transcriptome and selected blood biomarkers, CT measured small airway abnormality and parenchymal lung abnormalities in young adults.

Remote contacts will be used to keep the cohort engaged, update contact information and collect short-term follow-up information on the exposures and responses. We will endeavour to secure additional funding for future in-person exams and the evaluation of biospecimens.

\section{METHODS AND ANALYSIS \\ Study design}

The ALA-LHC is a longitudinal, multicentre cohort study that will enrol approximately 4000 participants aged 25-35 years old who do not have a diagnosis of severe lung disease recruited at participating research sites across the USA.

\section{Patient and public involvement}

The ALA-LHC was conceived and designed through close collaboration with the ALA, which regularly solicits input for its research programmes from participating patient advisory groups. Patients were not explicitly involved in the design of the ALA-LHC. The ALA will participate in the dissemination of results to the community of stakeholders interested in respiratory disease.

\section{Eligibility criteria}

Eligibility is deliberately broad and selected to be inclusive of young adults with a range of exposures that may influence lung health. However, individuals with established moderate-to-severe lung disease will not be enrolled. The detailed inclusion and exclusion criteria are provided in table 1 .

\section{Outcomes}

The primary outcome measures are as follows:

- In-person spirometry: Prebronchodilator lung function (forced expiratory volume in $1 \mathrm{~s}\left(\mathrm{FEV}_{1}\right)$, forced vital capacity (FVC) and peak flow) measured in accordance with the American Thoracic Society (ATS) /European Respiratory Society (ERS) standards and using United States National Health and Nutrition Examination Survey (NHANES) reference values. ${ }^{34}$

The primary outcome measures are as follows:

- Prebronchodilator FEV .

- Prebronchodilator FVC.

- ATS-Division of Lung Diseases (DLD) Respiratory Questionnaire: The ATS-DLD is a general respiratory symptom questionnaire. We will use the Lung Health 
Table 1 Inclusion and exclusion criteria

\begin{tabular}{ll}
\hline Inclusion & Exclusion \\
\hline Age 25-35 years old at the time of the enrolment & Severe asthma that is defined as any of the following: \\
& Current Global Initiative for Asthma Step 4 or higher therapy $^{27}$ \\
OR & Three or more unscheduled healthcare visits (provider/urgent \\
care/ER) for asthma in the past 12 months & OR \\
& One asthma hospitalisation in the past 12 months
\end{tabular}

Able to read and understand English or Spanish

History of any chronic lung disease other than asthma including but not limited to COPD, cystic fibrosis, pulmonary fibrosis, pulmonary hypertension or congenital lung disease

Has and is willing to share a social security number Current pregnancy

Resident (citizen or non-citizen) of the USA for at least 12 History of cancer other than non-melanoma skin cancer months prior to examination

Willing to provide contact information for at least two proxies who are likely to know the whereabouts and vital status of the participant
Diagnosed cardiovascular disease (ie, congenital heart disease and coronary heart disease)

History of cancer other than non-melanoma skin cancer Inability to comply with study procedures

Any condition that puts the participant at risk by participating in the study as deemed by study investigators (eg, serious respiratory illness requiring antibiotics or steroids or severe fever at the time of the study visit)

\section{Institutionalisation}

COPD, chronic obstructive pulmonary disease; ER, emergency room.

Study (LHS-III) modified version that includes information regarding occupational exposures and asthma:

- Any symptoms

- Individual symptoms

- CT measurements of small airway and parenchymal lung abnormalities will be made using both commercially available and open-source software (Chest Imaging Platform, an National Institutes of Health (NIH)-funded, open source platform, http://www. chestimagingplatform.org). ${ }^{5}$ The primary outcome measurements are as follows:

- Extent of parametric response map for functional small airways disease $\left(\mathrm{PRM}^{\mathrm{fSAD}}\right)$.

- Size of structural abnormalities (expressed as the percent of total lung with that classification (eg, $50 \%$ emphysema))

\section{Recruitment}

Participants will be recruited at each ALA Airways Clinical Research Centers (ACRC) site (box 1) through public advertising campaigns (digitally and through traditional media), public outreach, word of mouth and partnerships with community organisations. Information for potential and current participants will be posted on a publicly available study website hosted by the ALA, which will also organise a social media campaign.

The goal is to recruit a population that is balanced in terms of sex (target: 1/2 men and 1/2women) and educational attainment (target: $1 / 3$ high school education or less, $1 / 3$ some college and $1 / 3$ college degree or higher). Balancing recruitment by education as a proxy for socioeconomic status (SES) is based on data demonstrating individual behavioural factors (eg, occupation, smoking and physical activity) are associated with lung health and strongly correlated with SES. ${ }^{6}$ Individuals with lower levels of education are more likely to reside in lower-income communities, which will also encourage a distribution of environmental exposures. Although the recruitment targets are based on SES rather than race, we do anticipate recruiting a diverse population, with an expected racial distribution of approximately $60 \%$ Caucasian, 30\% African American, 7.5\% Asian, 2.5\% others and 25\% Hispanic/Latinx.

\section{Enrolment}

To initiate an in-person enrolment, a site will schedule an initial, in-person visit with the potential participant. All participants will provide written informed consent. The procedures and questionnaires are expected to take approximately 4 hours. Young adults often have conflicts (eg, need for childcare and limited time off) that would make it difficult to schedule such a large block of time. In order to make the initial assessment more feasible, the in-person visit may be broken up into several shorter visits with emphasis on completing as many of the assessments remotely as possible to reduce the in-person time. The 
Box 1 American Lung Association (ALA) Lung Health Cohort study sites and cores

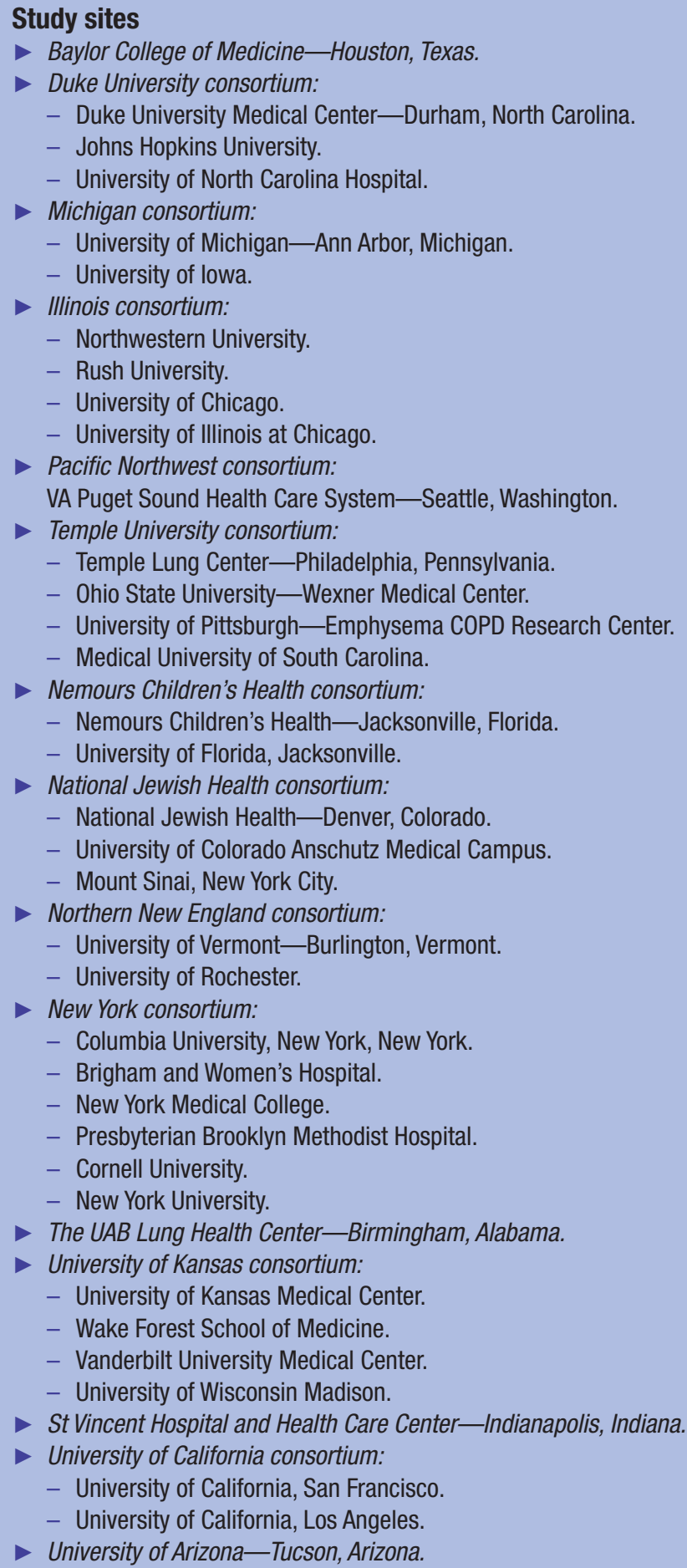

- Baylor College of Medicine-Houston, Texas.

- Duke University consortium:

- Duke University Medical Center-Durham, North Carolina.

- Johns Hopkins University.

- University of North Carolina Hospital.

- Michigan consortium:

- University of Michigan—Ann Arbor, Michigan.

- University of lowa.

- Illinois consortium:

- Northwestern University.

- Rush University.

- University of Chicago.

- University of Illinois at Chicago.

- Pacific Northwest consortium:

VA Puget Sound Health Care System-Seattle, Washington.

- Temple University consortium:

- Temple Lung Center-Philadelphia, Pennsylvania.

- Ohio State University-Wexner Medical Center.

- University of Pittsburgh-Emphysema COPD Research Center.

- Medical University of South Carolina.

- Nemours Children's Health consortium:

- Nemours Children's Health—Jacksonville, Florida.

- University of Florida, Jacksonville.

- National Jewish Health consortium:

- National Jewish Health-Denver, Colorado.

- University of Colorado Anschutz Medical Campus.

- Mount Sinai, New York City.

- Northern New England consortium:

- University of Vermont-Burlington, Vermont.

- University of Rochester.

- New York consortium:

- Columbia University, New York, New York.

- Brigham and Women's Hospital.

- New York Medical College.

- Presbyterian Brooklyn Methodist Hospital.

- Cornell University.

- New York University.

- The UAB Lung Health Center-Birmingham, Alabama.

- University of Kansas consortium:

- University of Kansas Medical Center.

- Wake Forest School of Medicine.

- Vanderbilt University Medical Center.

- University of Wisconsin Madison.

- St Vincent Hospital and Health Care Center_-Indianapolis, Indiana.

- University of California consortium:

- University of California, San Francisco.

- University of California, Los Angeles.

- University of Arizona - Tucson, Arizona.

\section{Study cores.}

- Administrative Core at Northwestern University.

- Actigraph Reading Center at Northwestern University.

- Data Coordinating Center at Johns Hopkins University.

- Spirometry Reading Center at Johns Hopkins University.

- Imaging Core at Brigham and Women's Health and University of Michigan.

Continued

\section{Box 1 Continued}

- ALA Airways Clinical Research Centers (ACRC) Biorepository at Nemours Children's Hospital.

- Environmental Exposure Core at Beth Israel Deaconess Medical Center.

first part of the in-person visit will focus on evaluating eligibility and obtaining informed consent for participation in the ALA-LHC. The procedures and key questionnaires relating to the primary aims of the study must be completed in person. The remaining questionnaires may be completed remotely.

\section{Study data collection}

Baseline assessment

The baseline assessment includes an in-person evaluation, wearable device monitoring of activity and remote contacts. Box 2 lists the procedures and questionnaire data collection for the baseline assessment. The total time allocated for the completion of all procedures and questionnaires is 4 hours. The goal is to collect all assessments within 1 month (28 days of enrolment); however, due to potential barriers to that collection schedule related to the ongoing COVID-19 pandemic, the permitted time interval for evaluations following initial enrolment may be longer.

\section{In-person activities}

Individuals will be consented, tested for eligibility and enrolled at the beginning of the in-person evaluation. All procedures except for the actigraphy monitoring and home spirometry will be performed in the clinic. In addition, key questionnaires will be completed during the in-person visit.

\section{In-home activities}

Additional in-home activities are planned to collect information on lung function, activity and vaping after the in-person visit. Each participant will be asked to complete a home spirometry session on five separate days over a 14-day period within a month of the in-person visit with coaching from a site coordinator via a telemedicine visit. In addition, each participant will be asked to wear an activity monitor for 1 week and then mail it back to the site. Those individuals who indicated that they have used e-cigarettes and vaping or heat-not-burn (HNB)/ heated tobacco devices will be asked to complete a 1-week daily diary recording the type and frequency of e-cigarette, vaping or $\mathrm{HNB}$ product use and any related symptoms.

\section{Remote contact activities}

Remote contacts will be used to facilitate the completion of the baseline assessment. Questionnaires that are not completed during the in-person visit will be completed remotely (eg, online by the participant using a link sent via email, text message, mail or phone). 


\section{Box 2 Activities and data collection}

\section{Consent}

\section{Eligibility evaluation}

\section{Enrolment}

\section{Procedures}

- Physical assessment (eg, height, weight, resting heart rate and blood pressure).

- Prebronchodilator spirometry at study site.

- Home spirometry (prebronchodilator).

- Pregnancy testing in participants of childbearing potential (prior to chest CT).

- Chest CT scan.

- Nasal epithelial lining fluid collection (Nasosorption strips).

- Nasal epithelial curettage.

- Phlebotomy (complete blood count, plasma, serum, RNA, DNA and SARS-CoV-2 serology).

- Urine collection.

- Actigraphy.

\section{Questionnaire domains}

- Social security number.

- Contact information for participant and two proxies.

- Home address history.

- Demographics (eg, date of birth, age, race, ethnicity, sex and birth, gender identity, education, employment status, income and health insurance coverage).

- Lung health:

- ATS-DLD Respiratory Questionnaire.

- Modified Rhinitis Symptom Utility Index. ${ }^{28}$

- Substance use (eg, tobacco, e-cigarette/vaping/heat-not-burn, alcohol and marijuana).

- Environmental and occupational exposures (eg, secondhand smoke, pets, mould and fumes).

- Medication use.

- Medical history (eg, disease diagnoses, allergies and pregnancy/ early life factors).

> Activity: - International Physical Assessment Questionnaire (short form). ${ }^{29}$

- Sleep: - Pittsburgh Sleep Quality Instrument. ${ }^{30}$

- Social elements (eg, neighbourhood safety, social roles and activities and global health).

- Nutrition.

- COVID-19 history and associated health behaviours (tobacco, ecigarettes, hand washing and social distancing).

\section{Patient logs}

Activity log.

Vaping diary.

\section{Remote follow-up}

During follow-up, participants will be contacted remotely (eg, via text message, email, mail or phone) every 3-6 months and asked to update their contact information and complete additional assessments related to the topics described above for the in-person visit (eg, lung health and behavioural habits) as well as health events (eg, urgent care visits, hospitalisation, vaccinations and diagnoses). In conjunction with each of the follow-up contacts, participants will be asked to schedule and complete a single, coached home spirometry session.

\section{Study procedures}

\section{Questionnaires}

Questionnaires may be completed in multiple ways. During the study visit, forms will be either directly data entered into the ALA-LHC REDCap data repository on tablets provided by the study or on paper forms, which will later be data entered by the site staff.

\section{Environmental evaluation}

The Environmental Exposure Core will use the lifetime address history of each participant to perform geocoding. The geocodes will be used to link relevant exposure data to each participant. Exposure data will include long-term averages of residential air pollution exposure (elemental carbon, organic carbon, nitrogen dioxide and sulfates), fine particulate matter $\left(\mathrm{PM}_{2.5}\right),{ }^{8-10}$ ozone, census-tract level indicators of neighbourhood socioeconomic position (median household income, median home value, measures of household crowding and distribution of education level) and weather data.

\section{Spirometry at the study site}

Prebronchodilator spirometry will be performed at the in-person baseline visit if local conditions and policies permit it. Each site will follow their institutional guidelines for conduct of research and COVID-19-related precautions and procedures and, if required at their local institution, institutional review board (IRB) approval.

Spirometry measurements taken at the site will adhere to ATS/ERS standards. ${ }^{3}$ Quality control, training and interpretation of results will be led by the Spirometry Reading Center at Johns Hopkins University. Spirometry measurements will include the FVC, $\mathrm{FEV}_{1}$ and $\mathrm{FEV}_{1} /$ FVC ratio. Only prebronchodilator spirometry will be performed (participants will not receive albuterol). Spirometry reference values will be those of Hankinson $e t$ al from NHANES. ${ }^{4}$ Spirometry sessions will be performed by a certified technician.

\section{Home spirometry}

All participants will be provided with a Spirobank Smart spirometer (MIR USA International Research), which is an Food and Drug Administration 510(k)-cleared device (K072979) for home use. After the in-person visit, participants will be asked to perform home spirometry on five separate days over a 14-day period within 1 month of the in-person visit. Therefore, participants will be asked to complete quarterly home spirometry sessions in conjunction with the quarterly contacts. The remote spirometry sessions will be coached by a certified technician using a video conference link.

\section{Chest CT acquisition}

Each participant will undergo CT imaging. CT scan will be acquired with multidetector CT scanners (64 detector channels) at full inspiration and expiratory effort using a 
low-dose protocol with dose modulation. Imaging reconstruction will use submillimetre slice thickness with four reconstructions per scan.

Standardised parameters will be developed for the CT scanner to be used at each site to ensure consistency of results. CT scanners at each site will be calibrated using a standard lung phantom prior to enrolling participants. All participants will be imaged in the supine body posture both at full inflation (approximating total lung capacity) and at the end of forcible exhalation (approximating residual volume).

Study measurements obtained on the CT scan will include features based on analyses of the lung parenchyma through the local histogram to determine lung inflammation, oedema, emphysema and interstitial changes as well as the $\mathrm{PRM}^{\mathrm{fSAD}}$ as has been used previously. ${ }^{11-16}$

\section{Nasal epithelial curettage}

Scraping of the nasal epithelium will be performed using five passes with the disposable plastic ASI Rhino-Pro nasal cytology curette from the inferior nasal turbine. Samples will be immediately frozen in RNA lysis buffer and stored at $-80^{\circ} \mathrm{C}$ until shipped to the repository for future processing.

\section{Nasal epithelial lining fluid}

For collection of nasal epithelial lining fluid, a thin absorbent, fibrous matrix designed to fit within the nasal passages (Mucosal Diagnostics Nasosorption FX.i) will be inserted into each nostril. Participants will be asked to pinch their nose closed for $1 \mathrm{~min}$ prior to removal of each strip. The two fibrous strips will be placed in separate tubes, capped and immediately frozen and stored at $-80^{\circ} \mathrm{C}$ until shipped to the repository.

\section{Phlebotomy}

Approximately $50 \mathrm{~mL}$ of blood will be collected by venipuncture from the arm or the back of the hand. One $4 \mathrm{~mL}$ tube will be transmitted to the local hospital laboratory without processing for measurement of complete blood count and chemistries. The remaining blood will be divided into $83250 \mathrm{uL}$ aliquots of serum and plasma for freezing and bulk storage pending shipping. One $2.5 \mathrm{~mL}$ PAXgene RNA tube will be used to collect blood for RNA analysis. Also, one lavender tube containing a remaining pellet after aliquoting plasma will be saved for DNA analysis. Both these tubes will be stored at -70 or $-80^{\circ} \mathrm{C}$ until shipped to the repository.

\section{Urine collection}

Participants will be instructed on how to obtain a clean catch urine specimen. A minimum of $20 \mathrm{~mL}$ will be collected from each participant. Urine will be aliquotted into $101 \mathrm{~mL}$ non-preserved aliquoting vials and placed at $-80^{\circ} \mathrm{C}$ for storage until shipment.

\section{Accelerometry}

At the conclusion of the in-person visit, participants will be given the ActiGraph GT3X. At the same time, they will be given a paper log to record when the device is removed and put back on and the intervals in which they were sleeping. Participants will be asked to wear the device on their non-dominant arm continuously for 24 hours a day over 7 days after leaving the site and to mail the device back to the clinic.

\section{COVID-19 exposure and history}

Participants will be given a questionnaire to assess selfreported history of COVID-19 and factors related to severity of disease. Exposure to SARS-CoV-2 will be assessed using serology testing.

\section{Data management}

\section{Data collection and transfer}

Data will be collected, stored and transferred in compliance with Health Insurance Portability and Accountability Act (HIPAA) Privacy and Security rules.

The primary mechanism for data collection and management in the ALA-LHC will be REDCap, a secure, web-based application hosted by the Data Coordinating Center (DCC) at Johns Hopkins University that can support direct electronic data entry or forms-based data capture in compliance with HIPAA Security Rule. ${ }^{17} 18$ The ALA-LHS REDCap Project will be managed by the DCC. The majority of enrolment and study data for the study visit will be directly entered to REDCap. Tablets will be provided to each site to facilitate real-time capture of data elements. However, paper versions of all instruments will be available as an alternative in case of connectivity issues, participant preference (in the completion of selfreported forms) or other barriers to direct data entry. In addition, each participant will be provided with a tablet to take home that can be used to complete forms remotely.

\section{Data sharing}

The ALA-ACRC DCC has developed and tested procedures for deidentification of personal health information (PHI) that meet the deidentification standard established by Section 164.514 of the HIPAA Privacy Rule. Deidentified datasets will be deposited to BioLINCC at scheduled milestones (eg, following completion of the baseline exam by all participants). Deidentified (coded) biospecimens collected in the study that are not analysed may be made available to qualified investigators along with a limited-use dataset in line with Office for Human Research Protections (OHRP) guidelines with a material transfer agreement and/or data-use agreement as applicable.

\section{Analyses}

Hypotheses

There are three primary hypotheses:

- Hypothesis 1: Air pollution and other inhalational exposures are associated with lower pulmonary function and more frequent respiratory symptoms, whereas physical activity is associated with higher function and less frequent symptoms.

- Hypothesis 2: CT-measured small airways and parenchymal lung abnormalities are associated with lower 
pulmonary function and more frequent respiratory symptoms in young adults.

- Hypothesis 3:

- The pattern of nasal epithelial gene expression is different in participants with CT-measured small airways and parenchymal lung abnormalities.

- Blood biomarkers previously associated with distinct chronic lung diseases such as Interstitial Lung Disease (ILD) and COPD (fibrinogen, $\mathrm{C}$ reactive protein, soluble receptor for advanced glycosylation end product, surfactant protein $\mathrm{D}$, club cell protein-16 and matrix metalloproteinases 7 and 9) are also associated with different CT patterns of lung abnormalities in young adults.

Hypotheses 1 and 2 will be addressed using data collected in the main ALA-LHC Study. Specimens will be collected to enable evaluation of Hypothesis 3 but will be deferred until additional funding is obtained.

\section{Sample size}

Detectable effect sizes were calculated assuming a sample size of 4000 (assuming 235 per site) and two-sided type I error rate of 5\% (unless otherwise specified). Simulations incorporating a random site effect produced similar results for variables used in prior ALA-ACRC studies due to low intraclass correlation coefficient among participants seen at the same site. Based on prior studies, ${ }^{19-21}$ we conservatively estimated that the SD of percentage of predicted $\mathrm{FEV}_{1}$ is to be 15. Data from the Framingham Study suggest that the SD of long $\mathrm{PM}_{2.5}$ is $1.24 \mu \mathrm{g} / \mathrm{m}^{3}$ with an IQR width (IQRw) of approximately 1.87 (ie, the SD for $\mathrm{PM}_{2.5}$ rescaled in units of IQRw is 0.66 ). We have $80 \%$ and $90 \%$ power to detect $0.98 \%$ and $1.13 \%$ decreases in percentage of predicted $\mathrm{FEV}_{1}$ of per IQRw increase in $\mathrm{PM}_{2.5}$ exposure, a clinically meaningful change as decreases of $1 \%-1.5 \%$ are associated with all cause mortality. ${ }^{22}$ In the Coronary Artery Risk Development in Young Adults Study, a cohort of 18-30 year olds enrolled in 1985; baseline symptom prevalence ranged from $10 \%$ (bronchitis) to $45 \%$ (wheeze). ${ }^{23}$ Assuming reference prevalence of $10 \%, 15 \%, 30 \%$ and $45 \%$, we have $90 \%$ power to detect an increase in the risk of symptoms to $12 \%, 17 \%, 33 \%$ and $48 \%$ (ie, OR: 1.19, 1.15, 1.12 and 1.11) per $I Q R w$ increase in $\mathrm{PM}_{2.5}$.

\section{Primary analyses}

The cross-sectional associations between lung health and modifiable risk factors (Hypothesis 1) and CT measurements (Hypothesis 2), as well as the association between CT measurements and biological markers (Hypothesis 3 ), will be assessed using mixed-effect models with a random intercept for study site. Robust SEs will be computed using statistical programme-based approaches when available and the bootstrap otherwise (eg, absolute risk differences). During model selection and formal testing, graphical (eg, residual plots and splines to assess linearity of the effect of continuous exposures) and analytical (eg, tests of normality) methods will be used to detect violations of modelling assumptions.

To evaluate Hypothesis 1, we will examine the crosssectional associations of modifiable risk factors (air pollution exposure, inhalational exposures and physical activity) with continuous ( $\mathrm{FEV}_{1}$ and $\left.\mathrm{FVC}\right)$ and binary (respiratory symptoms) measurements of lung health. In addition to the stratification variables, we will adjust all models for race/ethnicity; body mass index (BMI) (or height and weight); and, when appropriate, additional confounders. For environmental exposure, a centred exposure variable and a centre-specific mean exposure variable will be used to model within-centre and betweencentre contrasts, or alternatively, centre will be included as a categorical fixed effect to ensure that there is no residual confounding.

To evaluate Hypothesis 2, we will examine the crosssectional associations of continuous CT measurements of lung abnormalities (extent of PRM ${ }^{\mathrm{fSAD}}$ and percent lung abnormalities, log transformed if necessary) with continuous ( $\mathrm{FEV}_{1}$ and $\mathrm{FVC}$ ) and categorical (symptoms) measurements of lung health. In addition to the stratification variables, all models will include age, smoking and BMI (or height and weight).

To evaluate Hypothesis 3, we will model the association between blood biomarkers and continuous measures of lung abnormalities evaluated by CT (extent of PRM ${ }^{\mathrm{fSAD}}$ and percent lung abnormality) using the mixed-effect models described above. In addition to the stratification variables, all models will adjust for age, race/ethnicity, BMI (or height and weight) and smoking status.

\section{Subgroups}

Following the model of prior population studies, we will stratify recruitment to include an approximately equal number of men and women. This design enables us to test hypothesised risk factors separately in women and men. We anticipate that $40 \%$ of our sample will be non-white race and that $25 \%$ will be Hispanic/Latinx. Although we are not adequately powered to test for race/ethnicitybased interactions for most of our risk factors of interest, we will do stratified analyses by race/ethnicity that can be informative for describing whether the patterns of association within subgroups are consistent with those observed in the full population.

\section{Secondary analyses}

The analyses of continuous and binary secondary crosssectional outcomes will use the methods and modelling techniques described for the primary outcomes. Models will include adjustment for potential confounders, which may vary depending on the outcome of interest.

In addition to the cross-sectional data collected at the in-person visit, longitudinal data will be collected through the remote contacts. Continuous and binary outcomes will be modelled as described for the primary analyses with the addition of random effects and fixed effects (if appropriate) for time to account for repeated measurements within the 
same individual. Events will be evaluated in two wavs. Time to first event will be graphically explored using Kaplan-Meier curves, and comparisons will be made using Cox proportional hazards models for individual outcomes (eg, time to hospitalisation). Event rates and comparisons for repeated events (eg, rates of influenza vaccination) will be modelled using negative binomial regression to account for overdispersion, and the model will include a random effect to account for within-site correlation. ${ }^{24}$

\section{Missing data}

A variety of sensitivity analyses will be performed to determine the potential impact of missing data on our conclusions. 'Best' and 'worst' case single imputation techniques will be implemented to define the range of impact. In addition, the effect size that would need to be observed in the missing data in order to change inference will be computed. For a more sophisticated approach, a variety of tools including but not limited to multiple imputation and inverse probability weighting will be used. ${ }^{25} 26$

\section{Ethics and dissemination}

\section{Observational Study Monitoring Board (OSMB)}

The NHLBI appointed an OSMB who will review the charter, protocol and consent prior to the study initiation. Thereafter, the OSMB will meet annually with the research study team to review data regarding recruitment, data completeness, data quality and safety with additional meetings as needed. The OSMB will also review ancillary study proposals to evaluate the impact on participant burden and safety and the ability to achieve the ALA-LHC's objectives.

\section{Institutional review board}

IRB approval was obtained through a single IRB (Johns Hopkins Medicine). Participating study sites are expected to cede approval to the single IRB as per current NIH policy.

\section{Dissemination of data and biospecimens}

The ALA-ACRC DCC has developed and tested procedures for deidentification of PHI that meet the deidentification standard established by Section 164.514 of the HIPAA Privacy Rule. Deidentified datasets will be deposited to BioLINCC at scheduled milestones (eg, following completion of the baseline exam by all participants). Deidentified (coded) biospecimens collected in the study that are not analysed may be made available to qualified investigators along with a limited-use dataset in line with OHRP guidelines with a material transfer agreement and/or data-use agreement as applicable. The ALA-ACRC steering committee will review and, in consultation with LHC multiple principal investigator (mPI) team, approve requests for the use of biospecimens. A similar model will be used for sharing images. The ALA-ACRC DCC has extensive experience in preparation of limited-use datasets and maintains policies and model agreements for data-use agreements and material transfer agreements.

\section{Author affiliations}

${ }^{1}$ Pulmonary and Critical Care Medicine, Northwestern University Feinberg School of Medicine, Chicago, Illinois, USA

${ }^{2}$ Epidemiology, Johns Hopkins University Bloomberg School of Public Health, Baltimore, Maryland, USA

${ }^{3}$ Department of Epidemiology, Johns Hopkins University Bloomberg School of Public Health, Baltimore, Maryland, USA

${ }^{4}$ Medicine, Icahn School of Medicine at Mount Sinai, New York, New York, USA

${ }^{5}$ Pulmonary, Allergy and Critical Care Medicine, University of Alabama at

Birmingham, Birmingham, Alabama, USA

${ }^{6}$ Pulmonary and Critical Care Medicine, University of Michigan Michigan Medicine, Ann Arbor, Michigan, USA

${ }^{7}$ Radiology, Brigham and Women's Hospital, Boston, Massachusetts, USA

${ }^{8}$ Harvard Medical School, Boston, Massachusetts, USA

${ }^{9}$ Division of Pulmonary, Critical Care and Sleep Medicine, Beth Israel Deaconess Medical Center, Boston, Massachusetts, USA

${ }^{10}$ Environmental Health, Harvard University T H Chan School of Public Health, Boston, Massachusetts, USA

${ }^{11}$ Pulmonary and Critical Care, Brigham and Women's Hospital, Boston, Massachusetts, USA

${ }^{12}$ Preventive Medicine, Northwestern University Feinberg School of Medicine, Chicago, Illinois, USA

Acknowledgements American Lung Association Airways Clinical Research Centers, Baylor College of Medicine, Houston: Nicola Hanania, MD (principal investigator); David Wu, MD (coinvestigator); Dharani Narendra, MD (coinvestigator); Thomas Monaco, MD (coinvestigator); Mariana Sockrider, MD (coinvestigator); Mustafa Atik, MD (coordinator); Laura Bertrand, MD (coordinator). Brigham and Women's Hospital Asthma Research Center, Boston: Elliot Israel, MD (principal investigator); Victoria Forth, NP (coinvestigator); Angeles Cinelli, BA (coordinator). Columbia University Medical Center, New York: Emily DiMango, MD (principal investigator); Tarnjot Saroya (coordinator). Cornell University, New York: Robert J. Kaner, MD (principal investigator); Fernando J. Martinez, MD and MS (coinvestigator); Jamuna Krishnan, MD and MBA (coinvestigator); William Z. Zhang, MD (coinvestigator); Alicia J. Morris (coordinator); Lianne De La Cruz (coordinator); Keyla Ordonez (coordinator); Elizabeth Peters, RN and BSN (admin contact). Duke University Medical Center, Durham: Loretta G. Que, MD (principal investigator); Jason Lang, MD (principal investigator); Anne Mathews, MD (coinvestigator); Isaretta Riley, MD (coinvestigator); Antoinette M. Santoro, RRT (coordinator); Catherine Foss, RRT (coordinator). Johns Hopkins University, Baltimore: Nadia Hansel, MD/MPH (principal investigator); Ashraf Fawzy, MD (coinvestigator); Robert Wise, MD (coinvestigator); Meredith McCormack, MD/MHS (coinvestigator); Nirupama Putcha, MD/MHS (coinvestigator); Molly Lauver (coordinator); Karina Romero Rivero, MD (coordinator). Medical University of South Carolina, Charleston: Charlie Strange, MD (principal investigator); Margaret Hay, MD (coinvestigator); Danielle Woodford (coordinator); Kristen Neff (coordinator). Mount Sinai National Jewish Health Respiratory Institute, New York: Linda Rogers, MD (principal investigator); Sonali Bose, MD (coinvestigator); Chelsea Chung (coordinator). National Jewish Health Mount Sinai Respiratory Institute, Denver: Barry Make, MD (principal investigator); Juno Pak (lead coordinator). Nemours Children's Health, Jacksonville: Kathryn Blake, PharmD (principal investigator); Michelle Littlefield, BSN and RN (coordinator). New York Medical College, New York: Allen J. Dozor, MD, FCCP and FAAP (principal investigator); Alison T. Lennox, MD (principal investigator); Zachary Messer (coordinator). New York University, New York: Joan Reibman, MD (principal investigator); Rebecca Florsheim, MD (coinvestigator); Gail Schattner, MD (coinvestigator); Brittany Marti (coordinator); Tsering Tenzing, NP (coordinator). Northwestern University Feinberg School of Medicine, Chicago: Ravi Kalhan (principal investigator), Sharon Rosenberg (coinvestigator), Paul Reyfman (coinvestigator), Mercedes Carnethon (coinvestigator), Jenny Hixon (coordinator), Vanessa Garcia (coordinator). Ohio State University, Columbus: Philip Diaz, MD (principal investigator); Janice Drake (coordinator). Presbyterian Brooklyn Methodist Hospital, Brooklyn: Jeremy Weingarten, MD, MBA and MS (principal investigator); Christina Edwards, BS (coordinator). Rush University Medical Center, Chicago: James Moy, MD (principal investigator); Ben Hu (coordinator); Jun Fu (coordinator). St Vincent Health System, Indianapolis: Michael Busk, MD and MPH (principal investigator); Ellen Looney (coordinator). Temple University Health System Lung Center, Philadelphia: Gerard Criner, MD (principal investigator); Francine McGonagle (coordinator); Antoinette Santoro (coordinator). University of Alabama at Birmingham Lung Health Center, Birmingham: Mark Dransfield (principal investigator), Mike Wells (coinvestigator), Surya Bhatt (coinvestigator), Trisha Parekh (coinvestigator), Necole Seabron (coordinator), Renita Holmes (coordinator), Elizabeth Westfall 
(centre director). University of Arizona, Tucson: Lynn B. Gerald, PhD and MSPH (principal investigator); Dr Monica Kraft, MD (principal investigator); Chelsey Large, CRC (coordinator); Michele Simon, CRC (coordinator); Raymond Skeps, MS (coordinator). University of California, Los Angeles: Igor Barjaktarevic (principal investigator), Donald P Tashkin (coinvestigator), Russell Buhr (coinvestigator), Roslynn McGill (coordinator), Brian Jung (coordinator). University of California, San Francisco: Stephen C. Lazarus, MD (principal investigator); Julian Silva, MA (coordinator). University of Chicago Asthma and COPD Center, Chicago: Edward T. Naureckas, MD (principal investigator); Virginia Zagaja (coordinator). University of Colorado Anschutz Medical Campus, Aurora: Richard William Vandivier, MD (principal investigator); Fernando Holguin, MD and MPH (coinvestigator); Margaret Hope Cruse (coordinator); Vong Smith (asthma manager); Nancy Perez (coordinator); Kisori Thomas (coordinator); Leah Freid (coordinator); Fernando Diaz del Valle (coordinator). University of Florida, Jacksonville: James Cury (principal investigator), Vandana Seeram (coinvestigator), Fallon Wainwright (coordinator). University of Illinois Breathe Chicago Center, Chicago: Jerry A. Krishnan, MD and PhD (principal investigator); Lauren Castro (coordinator); Julie DeLisa (coordinator); Wendy Haas (coordinator); Jissell Torres (coordinator); Cindy Leman (director of admin operations); Namrita Berry (grants/contracts); Jennifer Sculley (design strategist). University of lowa, lowa City: Alejandro P Comellas (principal investigator), Kimberly Sprenger (coordinator), Eric Garcia (coordinator), Deborah O'Connell-Moore. University of Kansas Medical Center, Kansas City: Mario Castro, MD (principal investigator); Chase Hall, MD (coinvestigator); Scott Matson, MD (coinvestigator); Pam Kemp, PhD (coordinator); Vanessa Curtis, RRT (coordinator); Brynne Thompson, RN (coordinator); Jonathan Boomer, PhD (laboratory/biospecimen planning and processing); Josie Emery, BS (laboratory/biospecimen planning and processing); Elaine Worth, BS (laboratory/biospecimen planning and processing). University of Michigan, Ann Arbor: MeiLan Han, MD/MS (principal investigator); Alan Baptist, MD (coinvestigator); Wassim Labaki, MD (coinvestigator); Craig Galban, PhD (coinvestigator); Mary Kay Hamby (coordinator); Gretchen Bautista (project manager). University of North Carolina Hospital Obstructive Lung Diseases Clinical and Translational Research Center, Chapel Hill: M. Bradley Drummond, MD/MHS (principal investigator); Karen Hardy (coordinator); Andrea McDaniel-Harper (coordinator). University of Pittsburgh Emphysema/COPD Research Center, Pittsburgh: Frank C. Sciurba, MD and FCCP (principal investigator); Elizabeth Stempkowski, BA and CCRC (coordinator). University of Rochester Medical Center, Rochester: Sandhya Khurana, MD (principal investigator); Jessica Newcomb (research coordinator). University of Vermont Lung Center, Colchester: Charles Irvin, $\mathrm{PhD}$ (principal investigator); Anne Dixon, MA/BM/BCh (coinvestigator); Kevin Hodgdon RRT (coordinator). Pacific Northwest VA Puget Sound Health Care System, Seattle: Laura Feemster (principal investigator), David Au (coinvestigator), Emily Gleason (coordinator), Ed Udris (coordinator). University of Wisconsin Asthma, Allergy, and Pulmonary Research, Madison: Loren C. Denlinger, MD and PhD (principal investigator); Sean B. Fain, PhD (coinvestigator); Mark L. Schiebler, MD (coinvestigator); Lori Wollet, RN (coordinator); Mary Jo Jackson, RN (coordinator); Heather Floerke, BS (research specialist). Vanderbilt University Medical Center, Nashville: Katherine Cahill, MD (principal investigator); Leonard Bacharier, MD (coinvestigator); Julie Madison, RN (coordinator). Wake Forest Airways Clinical Research Center, Winston-Salem: Wendy C. Moore, MD (principal investigator); Anna Pippins, BS (coordinator); Isaac Deaton, BA, MA and EdS (coordinator). Chairman's Office University of Alabama, Birmingham: William C. Bailey, MD (chairman). LHC Protocol Committee: Ravi Kalhan (chair, contact PI) and Sonali Bose, Deb Brown and Mercedes R Carnethon, Mark T Dransfield, Laura Feemster, MeiLan Han, Heather Hazucha, Janet Holbrook, Marc Lenburg, Carly Petrusky, Lisa Postow, Susan Rappaport, Curt Reynolds, Mary Rice, Albert Rizzo, Alexandra Sierra, Elizabeth A Sugar, Lisa Viviano, George R Washko Jr, Robert A Wise. Administrative Core, Northwestern University: Ravi Kalhan (principal investigator), Mercedes Carnethon (coinvestigator), Jenny Hixon. Data Coordinating Center, Johns Hopkins University Center for Clinical Trials, Baltimore: Elizabeth A Sugar (principal investigator), Janet Holbrook (coinvestigator), Robert Wise (coinvestigator), Curt Reynolds (lead coordinator), Anne Casper (coordinator), Kathy Ewing (coordinator), Heather Hazucha (coordinator), Alexis Rea (coordinator), Gem Roy (coordinator), Emily Szilagyi (coordinator), Dave Shade (data system operator), Andie Lears (analyst), Jill Meinert (analyst), Marie Daniel (spirometry quality control expert). Imaging Core, Brigham and Women's Hospital, Boston: George Washko (principal investigator), Raul San Jose Estepar (coinvestigator), Pietro Nardelli (coinvestigator), Gonzalo Vegas Sanchez-Ferrero (coinvestigator), James Ross (coinvestigator), Samuel Ash (coinvestigator), Carrie Pistenmaa (coinvestigator), Stefanie Mason (coinvestigator), Alejandro Diaz (coinvestigator), Monica Iturrioz, Ruben San Jose Estepar. Spirometry Reading Center, Johns Hopkins University, Baltimore: Robert Wise (principal investigator), Marie Daniel (coinvestigator). ALA ACRC Biorepository, Nemours Children's Health, Jacksonville: Kathryn Blake (principal investigator), Ed Mougey (coinvestigator). Environmental Exposures Core, Beth Israel Deaconess Medical Center, Boston: Mary Rice (principal investigator), Murray Mittleman (coinvestigator). Project Office, American Lung Association, New York: Alexandra Sierra, MA; Susan Rappaport, MPH. Project Office, National Heart Lung and Blood Institute, Division of Lung Diseases, Washington DC: Tom Croxton (branch chief, airways biology and disease branch), Lisa Postow (program director, airway biology and disease branch), Lisa Viviano (clinical trials specialist, office of the director). Observational Data Safety Monitoring Board: Frank Gilliland (chair), Jonathan Goldin, Sharon Rounds, Mario Sims, Daniela Sotres-Alvarez.

Collaborators Elliot Israel; Emily Dimango; Robert J. Kaner; Loretta G. Que; Jason Lang; Nadia Hansel; Charlie Strange; Linda Rogers; Barry Make; Kathryn Blake; Allan J. Dozor; Joan Reibman; Philip Diaz; Jeremy Weingarten; James Moy; Michael Busk; Gerard Criner; Lynn B. Gerald; Monica Kraft; Igor Barjaktarevic; Stephen C. Lazarus; Edward T. Naureckas; Richard W. Vandivier; James Cury; Jerry A. Krishnan; Alejandro P. Comellas; Mario Castro; M. Bradley Drummond; Frank C. Sciurba; Sandhya Khurana; Charles Irvin; Laura Feemster; Loren C. Denlinger; Katherine Cahill; Wendy C. Moore; William C. Bailey; Janet Holbrook; Robert A. Wise; Murray Mittleman; Alexandra Sierra; Susan Rappaport; Albert Rizzo.

Contributors ES, MTD, MKH, RSJE, MBR, GRW, MC and RK contributed to the conception and design of the study. HH, JH, CR, SB, RSJE, MBR, GRW, ES, MC and RK developed the study protocol. PAR, ES and RK wrote the manuscript with input from all other authors.

Funding This work was supported by the National Heart, Lung, and Blood Institute grant number U01HL146408 and the American Lung Association.

Competing interests PAR reports personal fees from Medscape and Guidepoint, outside the submitted work. MTD reports personal fees and participation in contracted clinical trials from Boehringer Ingelheim, GlaxoSmithKline and AstraZeneca, outside the submitted work; participation in contracted clinical trials from Yungjin, PneumRx/BTG, Gala and Nuvaira, outside the submitted work; nonfinancial support, travel support and participation in contracted clinical trials from Pulmonx, outside the submitted work; personal fees from Quark Pharmaceuticals, Mereo, Teva and CSA Medical, outside the submitted work; and grants from ALA, outside the submitted work. MKH reports personal fees from Primelnc, during the conduct of the study; personal fees from AstraZeneca, Boehringer Ingelheim, GlaxoSmithKline, Cipla, Chiesi, Teva, Verona, Merck, Mylan and Sanofi, outside the submitted work; and research support from Novartis and Sunovion, outside the submitted work. RSJE is a founder and co-owner of Quantitative Imaging Solutions, outside the submitted work, and reports research support from Insmed and Lung Biotechnology, outside the submitted work; research support and personal fees from Boehringer Ingelheim, outside the submitted work; and personal fees from Chiesi and LeukoLabs, outside the submitted work. GRW is a founder and co-owner of Quantitative Imaging Solutions, outside the submitted work, and reports personal fees, research support and advisory board participation from Boehringer Ingelheim, outside the submitted work; personal fees and chairing of DSMB from PulmonX, outside the submitted work; personal fees and research support from Janssen Pharmaceuticals, outside the submitted work; personal fees from Novartis, outside the submitted work; and personal fees and advisory board participation from Vertex and CSL Behring, outside the submitted work, and GRW's spouse works for Biogen. RK reports research support from PneumRx (BTG) and Spiration, outside the submitted work; personal fees and research support from AstraZeneca and GlaxoSmithKline, outside the submitted work; and personal fees from Boehringer Ingelheim, CVS Caremark, Boston Scientific and Boston Consulting Group, outside the submitted work.

Patient and public involvement Patients and/or the public were not involved in the design, conduct, reporting or dissemination plans of this research.

Patient consent for publication Not required.

Provenance and peer review Not commissioned; peer reviewed for ethical and funding approval prior to submission.

Open access This is an open access article distributed in accordance with the Creative Commons Attribution Non Commercial (CC BY-NC 4.0) license, which permits others to distribute, remix, adapt, build upon this work non-commercially, and license their derivative works on different terms, provided the original work is properly cited, appropriate credit is given, any changes made indicated, and the use is non-commercial. See: http://creativecommons.org/licenses/by-nc/4.0/.

ORCID iDs

Paul A Reyfman http://orcid.org/0000-0002-6435-6001

Ravi Kalhan http://orcid.org/0000-0003-2443-0876 


\section{REFERENCES}

1 Reyfman PA, Washko GR, Dransfield MT, et al. Defining impaired respiratory health. A paradigm shift for pulmonary medicine. Am J Respir Crit Care Med 2018;198:440-6.

2 National Heart, Lung, and Blood Institute, National Institutes of Health, Department of Health and Human Services. New epidemiology cohort studies in heart, lung, blood, and sleep diseases and disorders (u01 - clinical trial not allowed), 2018. Available: https://grants.nih.gov/grants/guide/pa-files/PAR-18-577. htm

3 Miller MR, Hankinson J, Brusasco V, et al. Standardisation of spirometry. Eur Respir J 2005;26:319-38.

4 Hankinson JL, Odencrantz JR, Fedan KB. Spirometric reference values from a sample of the general U.S. population. Am J Respir Crit Care Med 1999;159:179-87.

5 Fedorov A, Beichel R, Kalpathy-Cramer J, et al. 3D slicer as an image computing platform for the quantitative imaging network. Magn Reson Imaging 2012;30:1323-41.

6 Cheng TL, Goodman E. Committee on pediatric R. race, ethnicity, and socioeconomic status in research on child health. Pediatrics 2015;135:e225-37.

7 Polak M, Szafraniec K, Kozela M, et al. Socioeconomic status and pulmonary function, transition from childhood to adulthood: crosssectional results from the Polish part of the HAPIEE study. BMJ Open 2019;9:e022638.

8 Di Q, Dominici F, Schwartz JD. Air pollution and mortality in the Medicare population. N Engl J Med 2017;377:2513-22.

9 Di Q, Rowland S, Koutrakis P, et al. A hybrid model for spatially and temporally resolved ozone exposures in the continental United States. J Air Waste Manag Assoc 2017;67:39-52.

10 Kloog I, Chudnovsky AA, Just AC, et al. A new hybrid spatiotemporal model for estimating daily multi-year PM2.5 concentrations across northeastern USA using high resolution aerosol optical depth data. Atmos Environ 2014;95:581-90.

11 Ash SY, Harmouche R, Ross JC, et al. The objective identification and quantification of interstitial lung abnormalities in smokers. Acad Radiol 2017;24:941-6.

12 Ash SY, Harmouche R, Ross JC, et al. Interstitial features at chest CT enhance the deleterious effects of emphysema in the COPDGene cohort. Radiology 2018;288:600-9.

13 Ash SY, Harmouche R, Vallejo DLL, et al. Densitometric and local histogram based analysis of computed tomography images in patients with idiopathic pulmonary fibrosis. Respir Res 2017;18:45.

14 Bhatt SP, Soler X, Wang X, et al. Association between functional small airway disease and FEV1 decline in chronic obstructive pulmonary disease. Am J Respir Crit Care Med 2016;194:178-84.
15 Galbán CJ, Han MK, Boes JL, et al. Computed tomography-based biomarker provides unique signature for diagnosis of COPD phenotypes and disease progression. Nat Med 2012;18:1711-5.

16 Kalhan R, Dransfield MT, Colangelo LA, et al. Respiratory symptoms in young adults and future lung disease. The cardia lung study. Am J Respir Crit Care Med 2018;197:1616-24.

17 Harris PA, Taylor R, Minor BL, et al. The REDCap Consortium: building an international community of software platform partners. $J$ Biomed Inform 2019;95:103208.

18 Harris PA, Taylor R, Thielke R, et al. Research electronic data capture (REDCap)-A metadata-driven methodology and workflow process for providing translational research informatics support. J Biomed Inform 2009;42:377-81.

19 Kalhan R, Arynchyn A, Colangelo LA, et al. Lung function in young adults predicts airflow obstruction 20 years later. Am J Med 2010;123:468.e1-468.e7.

20 Smith LJ, Kalhan R, Wise RA, et al. Effect of a soy isoflavone supplement on lung function and clinical outcomes in patients with poorly controlled asthma. JAMA 2015;313:2033-43.

21 American Lung Association Asthma Clinical Research Centers, Peters SP, Anthonisen N, et al. Randomized comparison of strategies for reducing treatment in mild persistent asthma. $N$ Engl J Med 2007;356:2027-39.

22 Sin DD, Wu L, Man SFP. The relationship between reduced lung function and cardiovascular mortality: a population-based study and a systematic review of the literature. Chest 2005;127:1952-9.

23 Kalhan R, Dransfield MT, Colangelo LA, et al. Respiratory symptoms in young adults and future lung disease. The cardia lung study. Am J Respir Crit Care Med 2018;197:1616-24.

24 Keene ON, Jones MRK, Lane PW, et al. Analysis of exacerbation rates in asthma and chronic obstructive pulmonary disease: example from the TRISTAN study. Pharm Stat 2007;6:89-97.

25 Daniels MH JW. Missing data in longitudinal studies; strategies for Bayesian modeling and sensitivity analysis. Boca Raton: Chapman \& Hall/CRC Press, 2008.

26 Little RJA, Rubin DB. Statistical analysis with missing data. 2nd ed. Hoboken, N.J: Wiley, 2002.

27 Global Initiative for Asthma. Global strategy for asthma management and prevention, 2021. Available: www.ginasthma.org

28 Togias A, Gergen PJ, Hu JW, et al. Rhinitis in children and adolescents with asthma: ubiquitous, difficult to control, and associated with asthma outcomes. J Allergy Clin Immunol 2019;143:1003-11.

29 Chu AHY, Ng SHX, Koh D, et al. Reliability and validity of the selfand Interviewer-Administered versions of the global physical activity questionnaire (GPAQ). PLoS One 2015;10:e0136944.

30 Buysse DJ, Reynolds CF, Monk TH, et al. The Pittsburgh sleep quality index: a new instrument for psychiatric practice and research. Psychiatry Res 1989;28:193-213. 